\title{
Internationales \\ Archiv für Sozialgeschichte der deutschen Literatur
}

Herausgegeben von

Georg Jäger, Dieter Langewiesche, Alberto Martino

20. Band 1995

2. Heft

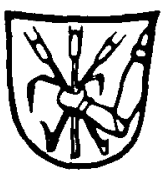

Max Niemeyer Verlag Tübingen 


\title{
Fortschrittsberichte und Forschungsdiskussion
}

\author{
Peter Strohschneider
}

\section{Die Zeichen der Mediävistik}

\begin{abstract}
Ein Diskussionsbeitrag zum Mittelalter-Entwurf in Peter Czerwinskis >Gegenwärtigkeit
\end{abstract}

Der Begriff der Gegenwärtigkeit bezieht bei Peter Czerwinski seine Bedeutung aus einer historischen Opposition zu dem des Zeichens. Er spricht diesem jede anthropologische Geltung $a b$ und zentriert solcherart den derzeit radikalsten Versuch, Mediävistik auf Alterität zu stellen. Der folgende Beitrag präsentiert und diskutiert dieses Konzept unter insbesondere methodologischen Gesichtspunkten.

In Peter Czerwinski's works, the concept of »Gegenwärtigkeit « derives its validity from the historical opposition to the concept of $»$ sign «. He denies the latter any anthropological validity and in this way produces the currently most radical attempt to base the study of mediaeval literature on alterity. This essay presents and discusses this concept, especially from a methodological point of view.

Die Schrift (wie wenn das möglich wäre) für einen Moment ausgeblendet, erschiene der Einband von Peter Czerwinskis "Gegenwärtigkeit «, ${ }^{1}$ ohne Bild oder Ornament, rein weiß: in einem Weiß, das nicht auf irgendetwas verweisen will etwa Thema, Inhalte, Diskurszusammenhänge des Buches -, was es nicht selbst wäre, in einem Weiß der reinen Gegenwärtigkeit. Nun ist indessen genau dies zugleich Thema und These des Werkes. Es handelt von einer Welt ohne Zeichendifferenz, einer Welt der Nicht-Repräsentation, der unmittelbaren Präsenz, der reflexionslosen Einheit, es handelt, kurz gesagt, von einer Welt der Gegenwärtigkeit, die es im europäischen Mittelalter ansiedelt. Verwiese darauf in gewissermaßen unvermittelter Referenzlosigkeit der rein weiße Buchumschlag, dann würden bereits an seiner materiellen Gestalt nicht allein Thema und These des Buches sichtbar, sondern in Gestalt dieser logischen Schleife auch seine kategorialen Kernprobleme: Die Referenzialität selbst des scheinbar Referenzlosen, damit die

${ }^{1}$ Peter Czerwinski: Gegenwärtigkcit. Simultane Räume und zyklische Zeiten, Formen von Regeneration und Genealogic im Mittelalter. Exempel einer Geschichte der Wahrnehmung II. München: Fink 1993. 
unauflösbare Differenz zwischen unserer und jeder fremden Welt der Nicht-Differenz und dieserart vor allen anderen jene Schwierigkeit, in den arbiträren Zeichenordnungen jeglicher Wissenschaftssprache - hier kehrt die ausgeblendete Schrift schon des Bucheinbands ins Spiel der Signifikanten zurück - von jenem ganz Anderen zu reden, das hier in den Blick geraten soll: sreinee, unvermittelte Gegenwärtigkeit als Ordnung einer fremden Welt.

Diese Rede setzt also sehr radikal an der Historizität nicht nur des Zeichenbegriffs, sondern des Zeichens selbst an. Sie kommt darum schwerlich umhin, sich mindestens tentativ von der Konventionalität literaturwissenschaftlicher Sprechhandlungen (deren Signifikate Signifikanten sind, welchen diese Sprechhandlungen ihre eigene Logik unterstellen) zu entfernen. Darin hat gewissermaßen seine Wahrheit, was bei einer Lektüre dieses Buches als erstes auffallen mag. Wilde Zitatcollagen, exuberante Fußnoten, das nicht zu bändigende Oszillieren der Argumentationslinien verstehen sich als Strategien, unter den Bedingungen einer wissenschaftlichen Abhandlung deren Genrenormen zugleich zu distanzieren, die 'logozentrische Rede fortzuführen und doch ihre Kolonisierungspotenzen gegenüber einer fremden Welt zu drosseln. Die aufstörenden, seine Lektüre erschwerenden sprachlichen Eigenheiten des Buches mögen so gesehen weniger stilistische Manierismen, als vielmehr Spuren systematischer Problemstellung sein.

Welche Hierarchie von ${ }^{-H a u p t}$ text und Fußnoten zum Beispiel jeweils gelten soll, ist in diesem Buch immer wieder nicht vorhersehbar. So muß die Lektüre in ständigem Hin und Her, Vor und Zurück zwischen den verschiedenen Textebenen springen, um überhaupt zu bemerken, daß dem ,Haupt text zuweilen allenfalls illustrierende Funktionen übertragen sind, daß umgekehrt wichtigste methodische Klärungen und Entscheidungen in scheinbaren Subtexten am Ende der Seite versteckt sein können - unter anderem auch diejenige, welche die skizzierte Schreibweise als ein programmatisches Verfahren mit dem Ziel reflektiert, »das Schreiben von den Fesseln der linearen Sukzession zu befreien « (S. 55 Anm. 77). Wohlkalkuliert scheint also ein Darstellungsverfahren, das in den Mikro- wie Makrostrukturen des Textes zunächst vielfach chaotisch und unübersichtlich wirkt; das den Lauf der Lektüre immer wieder auf frühere Etappen zirkulär zurückbiegt, das im Labyrinth der Phänomene und Reflexionen einen roten Faden der Argumentation gerade nicht vorzeigt (obgleich es ihn doch wohl gibt, vgl. unten II.), weil damit eben jene direktionale Linearität und Sukzessivität ins Recht gesetzt wären, deren mittelalterliche Negationen - Zyklik und Simultaneität - Czerwinski wahrnehmbar machen möchte. Wohlerwogen auch scheint es zu sein, wenn immer wieder die systematischen Hierarchien der Syntax durch Zusammenballungen von Sätzen und Satzgliedern suspendiert werden. Wie das aussieht, läßt sich nur an einer zusammenhängenden Textpartie zeigen. Ich wähle ein charakteristisches Beispiel aus dem Zentrum von Czerwinskis Argumentation. ${ }^{2}$

2 Der dabei wiederholt zitierte Text ist: Jochen Hörisch: Brot und Wein. Die Poesie des Abendmahls. Frankfurt/M. 1992. 
Wenn man aber davon ausgeht, daß der Aufgang einer Welt der Relation / der Bedeutung dem Ende einer des Sinns / der Substanz entspringt, dann ist die Möglichkeit des Wahrnehmens in Zeichen / Signifikanten / Symbolen historisch und kein Konstituens der Struktur menschlichen Denkens überhaupt.

,Daß zeitliches Sein und Dasein überhaupt bedeutsam sind, verdanken sie [...] den ontosemiologischen Leerstellen, die das verweisende und bedeutsame Spiel der Zeichen und dessen, was weltlich der Fall ist, allererst ermöglichen. Jedes Sinn-Integral, das keine Leerstellen im Reich der Zeichen und des Seins mehr zuließe, würde dieses Spiel sofort beenden.<

(Hörisch 1992, 276):

das wäre folglich keine anthropologische, sondern eine historische Bestimmung (die allein für die bürgerliche Gesellschaft Sinn hätte)?

Und an dieser Stelle nun eine Fußnote:

Nimmt man sie historisch, dann wäre auch der Zusammenhang zwischen Eucharistie und Geld, der bei Hörisch merkwürdig unscharf bleibt:

'Insofern markieren das Abendmahl, das Geld und die neuen Medien die drei großen

Formationen semontologischer bzw. ontosemiologischer Synthesis.< (Hörisch 1992, 13), aber:

`Geld und sogenannte `neue Medien [...] [als] funktionale Äquivalente von Brot und Wein< (a.a. O., 106),

klar: zwischen ihnen läge die vielleicht bedeutsamste Achse der Geschichte, jener Unterschied zwischen einer Wirklichkeit als Substanz und einer als Differenz, zwischen Sinn und Bedeutung, zwischen einer ontosemiologischen und einer nurmehr semiologischen Synthesis (deshalb halte ich es für unglücklich, von der Eucharistie als einem Medium [siche etwa a.a. O., 105] zu sprechen). [...]. (S. 87 mit Anm. 127)

Sprachformen dieser Art, die sich hier reich belegen lassen, zersetzen die üblichen syntaktischen Hierarchien, sie verschränken nicht in einem Satzgefüge integrierbare Elemente - was sekundäre Strategien ihrer Koordinierung hervortreibt, etwa Layout (Spatien, Einzüge, Schriftgrade) und Interpunktion ${ }^{3}-$, und es liegt nahe, sie als Aggregate zu verstehen. Insofern sind sie sprachliche Formen, die in ihrer Struktur wiederholen, was hier als konstitutiv für die mittelalterliche Welt der Gegenwärtigkeit behauptet wird: aggregative, also nicht-systematische Ordnungen. Nach den soeben schon hervorgehobenen Dichotomien von Linearität und Zyklik sowie von Sukzessivität und Simultaneität stellt sich damit - nur schon von ersten, ganz bei der scheinbaren Außenseite von Sprache und Argumentation verharrenden Beobachtungen her - nun ein dritter Binarismus ein, der von System und Aggregat. Es sind aber diese binären Terminologien, die Czerwinskis Mittelalter-Entwurf wesentlich organisieren (vgl. unten IV.). Ihm gegenüber ist also seine sprachliche Vermittlung nicht arbiträr.

Eines der - wenn es denn eines wäre - Risiken solchen Verfahrens liegt darin, daß die Kommunizierbarkeit dessen, was (und wie es) in Czerwinskis Entwurf der

${ }^{3}$ Durchgängig folgen auf Zitate zwei Satzzeichen, vor und nach dem Klammereinschub eines Quellennachweises, deren erstes die syntaktische Ordnung des zitierten, deren zweites diejenige des zitierenden Satzes markiert. 
Alterität der mittelalterlichen Welt gesagt ist, im Rahmen der üblichen fachlichen Diskurse prekär wird. Reaktionen, und die informellen deutlicher als die publizierten, schon auf den ersten Band der Exempel einer Geschichte der Wabrnebmung ${ }^{4}$ scheinen mir das zu belegen. Peter Czerwinski läuft dieses Risiko, so unterstelle ich, völlig bewußt, und es wird damit zugleich im Kalkül dieses Buches liegen, daß seine Diskussion in einer germanistischen Fachzeitschrift gar nichts anderes sein kann, als auch ein Akt seiner sozusagen disziplinären Disziplinierung.

II.

Ein solcher Versuch seiner zähmenden Rückholung in den Konventionsraum der Literaturwissenschaft beginnt dort, wo man von Czerwinskis Konzept referierend eine Vorstellung vermitteln will, seine kreisende Ausfaltung also einer linear sukzessiven Ordnung unterwirft. Zwar die Inszenierungsformen des Textes überspielen eine systematische Argumentationsstruktur, doch liegt sie selbstverständlich zugrunde. Präpariert man sie heraus, so zeigt sich, daß das Buch in spezifischer Weise sehr genau einlöst, was im Untertitel angekündigt ist. In seinen drei großen Kapiteln handelt es von der Simultaneität und aggregativen Zuordnung mittelalterlicher Räume (S. 11-189) und von dementsprechenden zyklischen Zeitstrukturen (S.191-256). Schließlich werden im umfänglichsten dritten Kapitel unterschiedliche kulturelle Objektivationen, u.a. Genealogien, volkssprachige Epik, Visionsliteratur, Goldbrakteaten oder Skatologien des 15. Jahrhunderts, als (insbesondere auch magische, rituelle) Aktualisierungen zyklischer Wiederholung, Wiederkehr, Regeneration gelesen. Wie nun dieser kategoriale Dreischritt von Raum, Zeit und Zyklus den Band und die totalisierende Vielfalt der zur Sprache kommenden Phänomene organisiert, das ist etwas genauer zu verfolgen.

Kapitel I schließt in seinem ersten Teil zunächst an einen schon in Der Glanz der Abstraktion eingeführten Zentralbegriff von Czerwinskis Mittelalterkonstruktion an, den der Aggregation: Strukturen der vermittlungslosen Zuordnung des - für uns - Inkongruenten, auf die dort von den Erzählformen volkssprachlicher Epik um 1200 her aufmerksam gemacht worden war, werden hier nun als überhaupt konstitutiv für mittelalterliche Raumkonstruktion dargestellt. Wiederum im Ausgang von narrativen Texten (Fortunatus), doch über sie hinausgehend, illustriert der Abschnitt die These, daß »nicht-bürgerliche Räume durch Bedeutung organisiert werden « - durch metaphysische Bedeutung sowohl als durch das gesellschaftliche Kraftfeld des adeligen Körpers -, und daß sie demnach »für uns zeitlich und örtlich nicht kongruente Teile einer Bedeutungseinheit, etwa der vita eines Heiligen, aggregativ nebeneinander enthalten können« (S. 52). Zu-

${ }^{4}$ Peter Czerwinski: Der Glanz der Abstraktion. Frühe Formen von Reflexivität im Mittelalter. Exempel einer Geschichte der Wahrnehmung [I]. Frankfurt/M. - New York 1989. 
gleich zeigt Czerwinski, »daß solche Räume uns flächig erscheinen müssen, weil sie unsere abstrakten, rein quantitativen Dimensionen und vor allem deren dritte, die Tiefe, nicht kennen (und nicht brauchen) (ebd.).

Der zweite Abschnitt (I.B.) differenziert den mittelalterlichen Aggregatraum als inhomogen und anisotrop (bes. S. $89 \mathrm{ff}$.) weiter aus, zeigt ihn als aus selbständigen >Blöcken $<$ aufgebaut, von Falten durchzogen und darin neuzeitlichen systematischen Räumen entgegengesetzt. Zum Aggregat gehört dabei, auch diese Kategorie ist im vorangegangenen Band bereits eingeführt worden, nicht eine kontinuierlich lineare Bewegungsform, sondern der 'Sprung ${ }^{5}$ als Vermittlungsmodus.

Der dritte Abschnitt des ersten Kapitels (I.C.) verfolgt sodann die Herausbildung eines homogenen, isotopen, systematischen Raumes. Beobachtungsfelder sind einerseits eine Gruppe von Renaissance-Bildern, auf welchen in Gestalt von Architekturteilen »das System [der Perspektive], das die Dinge dimensionieren soll, gleichsam selbst als Ding mit eigenem Recht aggregativ neben die Dinge gesetzt « werde (S. 96); der Terminus für solche »Erscheinung der Abstraktion in naturalförmiger Gestalt« lautet »Realabstraktion« (S. 147). Anderseits macht Czerwinski an Verkündigungsdarstellungen des 14.-16. Jahrhunderts evident, daß sie aus »besonderen, sinnerfüllten, qualitativen Räumen (S. 141) aggregiert sind. Auf einem Bild Tintorettos schließlich sieht er genau den Moment der Auflösung mittelalterlicher Raumaggregate gemalt, auf der Budapester Verkündigung El Grecos - und erst hier - sei Maria, mit der Taube des Hl. Geistes und dem Engel in einem einzigen, universalen Raum befindlich, die "Inkarnation des Allgemeinen, der transzendentalen Apperzeption« (S. 142f.).

Diese Deutung einmal unterstellt, läßt sich ihr freilich die Beobachtung anfügen, daß erst und genau in jenem Moment, für den El Grecos Bild hier in Anspruch genommen wird, der Verkündigungsengel von rechts kommt. Weil umgekehrt auf sämtlichen zuvor behandelten Bildern (wie auf der überwältigenden Mehrzahl aller Verkündigungs-Darstellungen aus Mittelalter und Renaissance) Gabriel aber von links kommt, ist es noch nicht ausgemacht, daß »sich die Frage nach >Leserichtungen` als tempozentrische Rejektion (S. 116) abtun läßt. Die regelmäßige Zuordnung der distinkten Räume auf den Verkündigungs-Bildern nach einem generellen Prinzip legt vielmehr die Frage nahe, ob nicht in der Ordnung des Aggregativen selbst Ansätze von Systematizität früh beobachtbar sind, mit welchen neben die Rezeptionsform der simultanen ${ }^{2}$ Schau ${ }^{6}$ jene der sukzessiv linearen Bild lektüre schon träte. Die Frage ist freilich zentral. Sie insinuiert die Hypothese (vgl. unten IV.3.), daß die Übergänge vom einen zum anderen Paradigma gleitender, also mit langen Phasen der Gleichzeitigkeit des Ungleichzeitigen

${ }^{5}$ S. 58 f. u. o.; der terminologische Apparat des Buches ist durch einen Index (S. $571-$ 575) insgesamt recht sorgfältig erschlossen, auf welchen ich hier zur Ergänzung meiner Nachweisungen ein für allemal verweise.

${ }^{6}$ Ein solches Lemma fehlt im Index; vgl. etwa S. 52, 95 Anm. 143, 104, 116, 156f., 217 Anm. 328. 
zu konzipieren wären. Nach dieser Vermutung wäre es nicht unproblematisch, auch noch den epistemischen Übergang vom Aggregativen zum Systematischen so wie es hier in der Diskussion von Tintorettos Verkündigung geschieht - nach der Logik des Sprunges zu denken.

Das ganz knappe Teilkapitel I.D. versucht sodann Ordnungen des Wissens, wie sie an Raumkonzepten rekonstruiert wurden, und solche der Gesellschaft aufeinander beziehbar zu machen: Wie für die bildhafte Konstitution des Raumes sei auch für die »Formen des Warentausches und der Geldbewegung um 1500 die Aggregation des konkreten Heterogenen (hier nun Gold) und des abstrakten Homogenen (Geld) bestimmend (S. 147). Das erste Kapitel schließt sodann mit kursorischen Bemerkungen (I.E.), welche die entwickelten Figuren insbesondere der räumlichen Aggregation und der Realabstraktion nun mit kartographischem Material, zumal am Prinzip der Portolane und an den hyperkomplexen Tafeln des Opicinus de Canistris, illustrieren.

Analog zur Differenzierung zweier Raumparadigmata im ersten kontrastiert das sehr viel knappere II. Kapitel einen modernen Begriff einer linearen, sukzessiv irreversibel verfließenden abstrakten Zeit mit seiner Negation: In nicht-bürgerlichen Kulturen sei Zeit charakteristischerweise zyklisch, nicht-linear und kontinuierlich, ihr Verlauf sei reversibel, sie sei nur als Aggregat bedeutungsgesättigter Zeitblöcke $z u$ verstehen und wie der Raum Falten, so werfe jene Zeit Blasen. Die einzelnen Abschnitte des Kapitels deklinieren diverse Konsequenzen dieser These durch: Sie sammeln nun aus der Geschichte der Zeittheorie Beispiele für Realabstraktionen (die jetzt Reinkorporationen heißen), also die Aggregation von systematischer, abstrakter und konkreter, aggregativer Zeit (II. B.); sie weisen für zyklische Zeit Kategorien wie Zufall und Entwicklung, das Auseinandertreten von Möglichkeit und Wirklichkeit (II.D.), aber auch Historizität und geschichtliche Faktizität als anachronistisch ab und bestimmen Ewigkeit unter ihren Bedingungen nicht als Unendlichkeit, sondern als umfassende Gegenwärtigkeit (II.C.). ${ }^{7}$ Den nächstliegenden und gewichtigsten Einwand gegen die Rekonstruktion mittelalterlicher Zeit als nichtlineare Zyklik, nämlich den Verweis auf die Konstruktion der Heilsgeschichte, diskutiert schließlich der Abschnitt II. E. Czerwinskis Lösung ist hier die Deutung

7 In diesem Zusammenhang geht Czerwinski S. $210 \mathrm{ff}$, auch auf die narrativen Strategien der Vorausdeutung und des Rückverweises ein. Er setzt dabei die Begriffe in distanzierende Anführungszeichen, denn: " Vorausdeutungen< und Rekapitulationen sind deutliche Hinweise darauf, daß die Begebenheiten eines Epos nicht anders als die Stationen eines Bildes auf irgendeine uns nicht mehr nachvollziehbare Weise nicht sukzessiv gelesen wurden, sondern tatsächlich simultan geschaut (S. 217 Anm. 228). Doch läßt sich mit gleichem Recht die Argumentation natürlich auch umkehren - und dies noch dann, wenn man einmal außer Acht läßt, daß das Epos und der Roman nicht so sehr gelesen oder geschaut, sondern vor allem gehört wurden: Daß in der Sukzessivität des Erzählaktes über Vorausdeutungen und Retrospektiven Simultaneität eigens hergestellt wird - und dies ja durchaus nicht stets so dicht wie etwa im Nibelungenlied, welches die Narratologie einer vergangenen Mündlichkeit im Schrifttext inszeniert -, dies könnte gerade indizieren, daß Simultaneität hier nicht (mehr unmittelbar) gegeben ist. 
eschatologischer Zeit als "Sonderfall zyklischen Denkens [...], bei dem es nur einen Zyklus gibt [...]«. (S. 256) Die vorausgesetzte Argumentation, wesentlich im Anschluß an Dieter Kartschoke ${ }^{8}$ vorgetragen, akzentuiert die stete Gegenwärtigkeit aller Stationen der Heils'geschichter, deren Logik »einer Einheit der Sätze nibil sub sole novum und semper novum « entspringe, welche allein dann einander nicht ausschlössen, wenn Zeit eben zyklisch gedacht werde (S. 254).

Solches »nicht-lineare[] Zeitdenken hieße besser Denken von Gegenwärtigkeit statt zyklisches Denken [...]« (S. 255). Dennoch ist es gerade dieser Begriff des Zyklus, den Czerwinski noch einmal benutzt, um den Zusammenhalt seines dritten, beinahe die Hälfte des ganzen Buches ausmachenden Kapitels zu markieren. Hatte das vorangegangene nach der simultanen Aggregativität der Räume die präsentische, nicht-lineare Zyklik der Zeiten als konstitutive Grundfigur nicht-bürgerlicher Welten etablieren wollen, so liegt es in der Konsequenz der insgesamt deduktiv geführten Argumentation, daß das Abschlußkapitel nun die These materialiter zu unterfüttern hat: Zyklische Repetition, Erneuerung im wiederholten Durchgang durch den Kreis des Jahres, des Lebens, Regeneration also ist darzutun als ein in diesen nicht-bürgerlichen Welten allgegenwärtiges, sie durchgängig bestimmendes Phänomen. In drei ausladenden Abschnitten sucht der Entwurf solche zyklischen Logiken zunächst in Genealogien auf: "genealogische Wahrnehmung erweist sich, weil sie bis zum mythischen Beginn der Dynastie zurückzufinden sucht, als Variante einer zyklischen Regenerations-Logik, denn die Gründertat des ersten Heroen besteht immer in einer Unterweltreise, (neue) Fruchtbarkeit/Herrschaft zu gewinnen « (S. 418). Die rituellen, kultischen Modi der Amorphose, welche Voraussetzung jener rites de passage am Eingang zur Gegenwelt, am Eintritt in zyklische Wiederholung seien (S. 351), zeigen die beiden letzten Abschnitte. In wuchernden Collagen von Quellenbelegen (in III.B. vor allem auch aus dem Bereich der Goldbrakteatenüberlieferung) wie von Forschungszitaten soll hier plausibilisiert werden, daß alle Formen des Zerreißens, Zerstückelns, Verschlingens, aber auch ihre Substitute, etwa Schmelzen oder Verbrennen (S. 382 ff.), Peinigung mit Wasser (S. 389 ff.), Kleidertausch (S. 439 ff.) oder Verhüllungen (S. $452 \mathrm{ff}$.) $\gg$ konstitutive, >positive Teile eines apokatastatischen Prozesses sind [...], magische rites de passage zur Überwindung des Spaltes zwischen altem, sterbendem und neuem, zu gebärendem Jahr, zwischen Diesseits und Jenseits, zwischen Jugend und Erwachsensein [...]« (S. 355). Der letzte Abschnitt schließlich interpretiert nach den Formen des Verschlingens nun auch konvulsivisches Sich-Ausschütten im Obszönen, im Skatologischen und im Gelächter, das Czerwinski im Begriff des Karnevals bündelt, als Regenerationsritual, ${ }^{9}$ um solcherart die Omnipräsenz des Regenerativen in allen vorbürgerlichen und nicht-bürgerli-

${ }^{8}$ Vgl. Dieter Kartschoke: Nibil sub sole novum? Zur Auslegungsgeschichte von Eccl. I. 10. In: Christoph Gerhardt [u. a.] (Hg.): Geschichtsbewußtsein in der deutschen Literatur des Mittelalters. Tübingen 1985, S. 175-188.

" oder Reflex davon: S. 473 Anm. 860. 
chen Welten zu erweisen. - Ich gestehe meinen Eindruck, daß zumal in diesem zweiten Teil des Buches die Ekstase des Materialverschlingens und Belegausschüttens sich zuweilen gegenüber methodisch noch kontrollierbarem, auch transparentem Argumentieren - welches sie ja nicht ersetzen kann - verselbständige. Dessen Struktur insbesondere ist auf lange hin kaum genauer zu erkennen als in der hier angedeuteten kausalen Ordnung, wonach es zunächst um Genealogie, sodann um Rituale prägenerativer Amorphose, schließlich um die späten Reflexe nicht-bürgerlich - so die These - ubiquitärer Regenerationsrituale auf der Schwelle zur neuen, linearen, nicht-zyklischen, homogenen Zeit geht. ${ }^{10}$

III.

Solches sind nun freilich, worauf es doch ankäme, noch keine systematischen Einwände gegen Peter Czerwinskis Mittelalter-Konzeption. Daß das Rauschen der Zitate die argumentative Rede zuweilen völlig übertöne, besagt gegen diese ja noch wenig. Gewichtiger wäre die Behauptung, daß dieser zweite Band der Exempel einer Geschichte der Wabrnehmung die Begründungsprobleme des vorangegangenen argumentativ weiterzuverfolgen gar nicht unternehme, daß er sie vielmehr hinter der Fülle des Materials zum Vergessen zu bringen trachte - polemisch verkürzt gesagt: daß er im. Widerspruch zur beanspruchten Modellhaftigkeit (S. 8), doch im Einklang mit der theoretischen Grundfigur des Entwurfes darauf abziele, die Rationalität wissenschaftlichen Argumentierens durch unmittelbare Evidenz - vielmehr durch deren Anschein - zu ersetzen. Noch einmal von jener Schreibweise her, »deren Problem es ist [...], zwischen Essay und Monomanie einherzuschwanken ", "wäre mit einer solchen Behauptung - und ich bin ungewiß, ob sie sich wirklich begründen ließe - nun auch dorthin der Weg gebahnt, wo wohl eines der systematischen Zentralprobleme von Czerwinskis Projekt liegt. In ihm artikuliert sich nämlich eine von unterschiedlichen Theoriebeständen poststrukturalistischer Provenienz eher inspirierte denn begründete Kritik an der slogozentrischen< Rationalität mediävistischer Forschung: Sie würde nun einerseits auf der Ebene der Darstellung auch zu einer Distanznahme gegenüber den dort geltenden Argumentationsstandards führen; gegen die Mittelbarkeit wissenschaftlichen Argumentierens wird öfters die fingierte Unmittelbarkeit des Zitierens und Montierens von 'Quellen< gesetzt. Anderseits aber und im Widerspruch dazu verlängert Czerwinskis Projekt zugleich und explizit eine geschichtsphilosophische Tradition (S.8 Anm.7), deren Ontologie und Totalisierungsan-

${ }^{10}$ Daß dem zugleich etwa eine ausgearbeitete Theorie des höfisches Romans, der hier territorialhöfisches Epos heißt, eingeschrieben ist (III. A.), wird erst bei der kontinuierlichen Durcharbeitung des ganzen Buches bemerklich.

${ }^{11}$ Der Glanz der Abstraktion, S. 15 Anm. 6: Czerwinski hat in der Regel längst antizipiert, was Kritiker ihm vorrechnen mögen, doch bleibt solche Antizipation nicht selten ohne argumentative Folgen. 
sprüchen der Poststrukturalismus gerade den Boden zu entziehen sich anschickte, eine slogozentrische Geschichtsphilosophie, die dem Logozentrismus und den mit ihm entstandenen Differenzen von Geist und Natur, Bewußtsein und Sein, Signifikant und Signifikat etc. eine Welt primordialer Einheit, Unmittelbarkeit und Gegenwärtigkeit historisch voraussetzt. Darin liegt in der Tat das schon im ersten Band sich abzeichnende ${ }^{12}$ theoretische Kernproblem der Exempel einer Geschichte der Wabrnebmung. Mit Blick auf solchen Widerspruch, daß einerseits Logozentrismus-Kritik und anderseits ein nur präsenzontologisch und damit logozentrisch fundierbares Unmittelbarkeits-Paradigma hier einander gegenseitig stützen müssen, mag die von Otto Gerhard Oexle in dieser Zeitschrift (IASL 20 [1995] 1. Heft, S. 203-208) formulierte Kritik selbst in ihrer polemischen Zuschärfung vielleicht nicht ohne Plausibilität sein, welche Czerwinskis Entwurf einer Mittelalter-Nostalgie zuschlägt, die sich »nach Befreiung von den Zumutungen der Moderne« (ebd., S. 208) sehnt.

Viele Einwände auch im Einzelnen ließen sich wohl aus dem skizzierten Widerspruch ableiten oder als sein vorab zu entrichtender Preis namhaft machen: So die Indifferenz gegenüber den Diskurszusammenhängen der lateinisch-klerikalen, schriftsprachlichen Gelehrtenkultur des Mittelalters ${ }^{13}$ oder die Ausblendung jeder Differenz zwischen einer Geschichte der Wahrnehmung und einer Geschichte der Reflexion über Wahrnehmung (weil eben für die nicht-bürgerliche Welt Reflexion und Wahrnehmung als ungeschieden unterstellt werden: woraus indes keineswegs folgt, daß sie es auch für die wissenschaftliche Rekonstruktion sein dürften). Demgemäß wird hier eine Strategie des einzigartig radikalen Wörtlichnehmens der Überlieferung verfolgt, welche - vor allem in den Textinterpretationen schon von Der Glanz der Abstraktion - in der konkreten Arbeit am Material vielfältig Neues sichtbar werden läßt, welche aber zugleich als generalisiertes hermeneutisches Prinzip aporetisch wird: Nach Czerwinski hätten wir »methodisch gar keine andere Wahl, als den Text, das Bild exakt so zu nehmen, wie sie dastehen." (S. 79) Damit soll im Zusammenhang der Argumentation gesagt sein, daß, was die Texte und Bilder zeigen, in unmittelbarer Gegenwärtigkeit die historische Realität shinter< den Texten und Bildern ssei<, also nicht zugleich auch Effekt von Darstellungsstrategien. Poetologien, Narratologien usw. kann es in der Welt totaler Präsenz a priori nicht geben. Indes besagt der zitierte Satz genau besehen etwas ganz anderes: Text und Bild >stehen< zunächst ja nicht einfach >da<, sondern wir stellen sie hin. Alles Überlieferte ist immer schon ein Vermitteltes. Dies »exakt so zu nehmen", wie es dasteht hieße darum, Text und Bild just als Text und Bild zu nehmen und nicht als das, was es für uns jedenfalls nicht mehr gibt: die >unvermittelte Präsenz< des Vertexteten und des Abgebildeten. Es ist genau dieser Sinn eines seiner zentralen methodischen Sätze, den Czerwinskis Mediävistik-Entwurf zu

12 Vgl. dazu auch die Rezensionen von Ralf Simon in: Arbitrium 9 (1991) S. 153-157; Jan-Dirk Müller in: PBB 114 (1992) S. 509-515 (hier v. a. S. 509 f.).

13 Vgl. Müller (Anm. 12), S. 512 f. 
negieren versucht. Selbst wenn indes Text und Bild einmal reine Gegenwärtigkeit gewesen sein sollten, dann bliebe doch die Frage offen, wie es historisch überhaupt zu ihnen hat kommen können, warum sich eine Welt der Gegenwärtigkeit in Texten und Bildern hätte verdoppeln sollen, wenn dort nichts anderes der Fall wäre, als was ohnedies schon der Fall ist.

$\mathrm{Zu}$ den Kosten des angedeuteten Widerspruchs zwischen poststrukturalistisch und geschichtsphilosophisch inspirierten Theoriefiguren gehört weiterhin auch jene fast vollständige Nivellierung aller nicht-bürgerlichen Kulturen, welche die Herausforderungen der Ethnologie nicht als methodologische begreift, sondern einfach inhaltlich nimmt: Mittelalterliche Kulturen erscheinen ihr als eben jene archaischen Welten der "reflexionslose[n] Einheit der vollen sozialen Körper" (S. 498), welche geschichtsphilosophisch vorausgesetzt wurden. Deswegen kann es auch geschehen (S. 307), daß etwa ein Zitat von Jan Assmann über den altägyptischen Zeitbegriff ganz unmittelbar als Interpretation des Iwein-Prologs von Hartmann von Aue fungiert. ${ }^{14}$

An solchen und vergleichbaren Stellen - und ganz selten sind sie nicht - hält es schwer nicht polemisch zu reagieren. Czerwinski hat es sich, aller an sein Projekt gewendeten Mühe ungeachtet, nicht nur mit dessen theoretischen Grundlagen zu leicht gemacht, sondern auch mit Durchführungen im Einzelnen. Vor allem aber hat er es damit seinen disziplinären Kritikern zu leicht gemacht. Wie sehr, das möchte ich hier in einer kleinen referierenden Skizze des Abschnitts II.A. »Blasen und Blöcke« (S. 193-221) verdeutlichen.

Ausgangspunkt ist eine Kategorisierung sdes< modernen Begriffs der Zeit »von der Newtonschen Mechanik über die Thermodynamik bis zur Quantentheorie (S. 194) als linear, sukzessiv, irreversibel und abstrakt (S. 193-197). In enger Anlehnung an eine Untersuchung von Jan Assmann wird dem im zweiten Argumentationsschritt als Negation der altägyptische Begriff einer zyklischen, nicht-linearen, reversiblen, oszillierenden, damit Fortschritt und Geschichte ausschließenden Zeit gegenübergestellt (S. 198-201). Hieran schließt die Setzung an, dieser Begriff lasse sich »sicher verallgemeinern", er fasse »die Form der Zeitwahrnehmung in vielen nicht-bürgerlichen Kulturen [...] (S. 199f.). Auf diese Setzung folgt der begründungslose, weil unzulässige Umkehrschluß, »Zeit san sich««, all das, was der altägyptische Zeitbegriff ausschließt, sei »also [...] eine historisch sehr junge

${ }^{14}$ Genauso wird immer wieder Zitaten aus ethnologisch-anthropologischer Forschungsliteratur plausibilisierende Geltung im Rahmen mediävistischer Darstellungszusammenhänge zugemutet; vgl. etwa S. $60 \mathrm{f}$., $89 \mathrm{f}$., $218 \mathrm{ff}$., $259 \mathrm{f}$., $301 \mathrm{f}$. mit Anm. 506, usw. Das heißt zugleich, daß der Einwand, der $\approx$ geschichtsphilosophische Entwurf [werde] auf problematische Weise historisch-chronologisch konkretisiert« (Müller [Anm. 12], S. 510), gegenüber Czerwinskis zweitem Buch an Gewicht verliert: Nur partienweise noch handelt es vom deutschen Mittelalter, vielmehr werden die Strukturen jener Welt, die hier vorwiegend nicht-bürgetlich heißt, derart generalisiert, daß viele Aussagen eine im konventionellen Sinne historische Bezeichnungsfähigkeit weitgehend verlieren (vgl. auch unten Anm. 22). 
Wahrnehmungsform, die « - wieder eine Setzung - "im Zusammenhang steht mit dem tatsächlichen Heraustreten aus der Einheit eines in die jeweiligen Besonderungen des Handelns und der Dinge versenkten Lebens [...]« (S. 201 f.). Zeit als abstrakte gehöre, davon dürfe man ausgehen [!], mit anderen Abstraktionen wie etwa dem Geld historisch zusammen (S. 203), und gleichermaßen gelte [!] solche Homologie auch für die Strukturen von Zeit und Raum in nicht-bürgerlichen Welten. Dies ist die ganze Logik einer Argumentation, an deren Ende die bei Assmann gefundenen Bestimmungen des altägyptischen Zeitbegriffs als konstitutiv für "mittelalterliche Wahrnehmung « beansprucht werden (ebd.). Die anschlieBenden Seiten können sich folglich dabei begnügen, mit Beispielen aus ethnologischen Untersuchungen wie aus mittelalterlichen Texten die deduzierten Kategorien zu illustrieren.

Dies mag für hier genügen. Ich will nämlich nicht etwa bestreiten, daß mittelalterlicher Wahrnehmung Zeit unter bestimmten Bedingungen etwa zyklisch oder nicht-abstrakt, sondern bedeutungsgesättigt gewesen sein mochte. Wohl aber sollte hier deutlich geworden sein, wie man wohl Argumentationsstrukturen keinesfalls aufbauen dürfte, wollte man provozierenden Thesen in fachwissenschaftlichen Diskussionen, mediävistischen etwa, ihren Platz sichern.

IV.

Peter Czerwinski macht es also sich und seinen Kritikern sowohl auf der Ebene genereller theoretischer Begründungszusammenhänge wie auf jener von Detailargumentationen immer wieder viel zu leicht. Doch sollte man es sich nicht so einfach machen, es bei dieser Feststellung sein Bewenden haben zu lassen, denn zwischen diesen beiden Ebenen des Textes gibt es Zwischenstufen. Schließlich ist man keineswegs genötigt, dieses Buch von den Eigenwilligkeiten seiner Darstellungs- und Begründungsstrategien her zu lesen. Man muß es auch nicht allein von seinem Versuch (?) einer Vermittlung geschichtsphilosophischer und poststrukturalistischer Theoreme her wahrnehmen; und ich bin ganz unsicher, ob dies schon bedeuten würde, den Entwurf gegen den Strich zu lesen. Weiterführend scheint es mir vielmehr, sich - aller nötigen Kritik eingedenk - an das Programm einer radikalen Historisierung zu halten, welches Czerwinskis Projekt trägt und welches mit dem vorliegenden Buch und seinem Vorgänger zwar noch nicht abgegolten, aber doch auch nicht erledigt ist. Es ist der gegenwärtig radikalste, daher wie ich zu zeigen mich bemühte - bis auf die Ebene seiner eigenen Sprachlichkeit durchschlagende Versuch, Mediävistik auf Alterität zu stellen.

Gegen die kolonisierende Vereinnahmung des total fremden Mittelalters ist Czerwinskis Projekt zuallererst gerichtet, und es findet den kolonialistischen Zugriff immer wieder vor allem in den Begriffen der mediävistischen Sprache, zumal in dem dort selbstverständlichsten, im Begriff des Zeichens. Es wird in diesem Konzept "(nach wie vor) mit der Möglichkeit einer historischen Alterität von 
Wahrnehmungsstrukturen gerechnet (und folglich der Satz bestritten: >Das Zeichen und die Göttlichkeit sind am gleichen Ort und zur gleichen Stunde geboren. ${ }^{15}$ (Im übrigen ist der Begriff >Präsenz< durch den der >Gegenwärtigkeit< ersetzt, denn der läßt sich, weil weniger an ihm hängt, leichter handhaben.) « Und an dieser Stelle der Fußnote nun der zentrale programmatische Satz: »Die historische Dimension des Begriffs der Gegenwärtigkeit liegt also in seiner Opposition zu dem des Zeichens, insofern durch diese Entgegensetzung dem Zeichen eine anthropologische Geltung abgesprochen wird (S. 7 Anm. 4).

Es könnte sein, daß weniger in einer Verquickung geschichtsphilosophischer mit poststrukturalistischen Theoriebeständen noch in der nostalgischen Sehnsucht nach Entlastung von den Zumutungen der Moderne der Ausgangspunkt von Peter Czerwinskis Mediävistik-Projekt liegt, daß vielmehr sein eigentlicher (und nach meiner Meinung für die Erforschung des Mittelaiters auch außerordentlich wichtiger) Impuls etwa an dieser Stelle formuliert ist - und damit zugleich die methodisch wie sachlich problematische Strategie seiner Verarbeitung. Ich versuche dies in vier Interpretationsschritten noch einmal systematisch zu entwickeln.

1. Historizität der Zeichen: Dieser Impuls ist die Historisierung nicht bloß des Zeichenbegriffs (daß der seine komplizierte Geschichte hat, weiß man), sondern seines Referenten, ist das Ausloten der Frage, inwiefern Signifikanten unter Umständen als metonymische oder analoge nicht nur theoretisiert wurden, sondern ssubstanzielk - mit ihren Signifikaten Beziehungen der Ähnlichkeit, der Identität unterhielten (dann also keine differenziellen >Beziehungen< unterhielten, keine ZZeichen waren) - und wie eine Kultur verfaßt wäre, in welcher solches konstitutiv der Fall sein konnte.

Nun gibt es hinlänglichen Anlaß zu der Annahme, das europäische Mittelalter sei eine solche Kultur gewesen. Sie sind ja überliefert: die Diskurse - nur zum Beispiel - über die zwei Körper des Königs; ${ }^{16}$ über den ontologischen Nexus von verbum und res; über die Eucharistie; über die Wahrnehmbarkeit der präsenten Absenz Christi zwischen Auferstehung und Himmelfahrt; über die Achiropiiten, ${ }^{17}$ die keine Ab-Bilder sind, keine Repräsentationen, sondern die wunder-

15 Dieses Zitat aus Jacques Derrida, Grammatologie. Frankfurt/M. 1983, S. 28. - Dem deduktiven Duktus des Buches gemäß wird historische Alterität von Wahrnehmungsstrukturen, so versteht sich, freilich gerade nicht als Möglichkeit unterstellt, sondern als Gegebenheit vorausgesetzt: Die "Differenz von Wirklichkeit und Möglichkeit«, welche das Buch seinem Gegenstand bestreitet (S. 246), kommt ihm selbst immer wieder abhanden (auch am oben referierten Abschnitt II. A. war das etwa zu beobachten).

16 Vgl. Ernst H. Kantorowicz, The King's Two Bodies. A Study in Medieval Political Theology. Princcton, NJ 1957; Hasso Hoffmann, Repräsentation. Studien zur Wortund Begriffsgeschichte von der Antike bis ins 19. Jahrhundert. (Schriften zur Verfassungsgeschichte 22) Berlin 1974.

17 Das sind die smagischen ungemalten Christus rbilder (vgl. Ernst von Dobschütz, Christusbilder, Untersuchungen zur christlichen Legende. [Texte und Untersuchungen zur Geschichte der altchristlichen Litcratur, N.F. 3] Leipzig 1899; Hans Belting: Bild und Kult. Eine Geschichte des Bildes vor dem Zeitalter der Kunst. München ${ }^{2} 1991$, 
mächtige >Gegenwärtigkeit< des Heilands selbst. Und mit Czerwinski halte ich es keineswegs für ausgemacht, daß solche Diskurse ohne Entsprechung in der Geschichte der Wahrnehmung geblieben seien. Konsensfähig mag darüberhinaus die Einschätzung sein, es sei das hierin implizierte historische und theoretische Problempotential in den Mittelalterwissenschaften schwerlich schon hinlänglich ernst genommen worden. ${ }^{18}$ In diesem Zusammenhang nun - der hier nur eben als solcher benannt werden konnte - ist der Vorstoß Czerwinskis, selbst in seiner monoman wirkenden Intransingenz, entschieden wichtig. Und dies nicht nur, weil es in der gegenwärtigen Forschungslandschaft kaum vergleichbare Alternativen gäbe: Die Mittelalterwissenschaften brauchen diese Provokation.

2. Zeichendifferenz als historisch sekundäre: Entscheidend ist aber auch, daß dieser Vorstoß zur Historisierung der Zeichen erst in seinem geschichtsphilosophischen Horizont spezifische Kontur gewinnt: Zeichendifferenz wird nicht einfach als etwas historisch Kontingentes, sie wird als etwas historisch Sekundäres aufgefaßt. Czerwinski setzt nicht Koexistenz (Simultaneität) verschiedener Möglichkeiten von Präsenz und Repräsentation voraus, sondern Chronologie (Sukzession). Er unterstellt, daß es historisch vor der Welt der Zeichen eine solche der Nicht-Differenz, eben der Gegenwärtigkeit gegeben habe (vgl. v. a. auch S. 269 ff.): »Wenn man aber davon ausgeht, daß der Aufgang einer Welt der Relation / der Bedeutung dem Ende einer [Welt] des Sinns / der Substanz entspringt, dann ist die Möglichkeit des Wahrnehmens in Zeichen / Signifikanten / Symbolen historisch und kein Konstituens der Struktur menschlichen Denkens überhaupt « (S. 87).

Wie sich diese konditionale Setzung einstellt, welche hier kritisch ist, läßt sich etwa an Bemerkungen gerade zur Eucharistie beobachten: »das Heilige bleibt als Ding heilig, die Hostie ist hier der Leib Christi und bedeutet ihn nicht (S. 86). Daraus folgert Czerwinski: »Folglich ist es problematisch, vom Mittelalter als einer Welt der Symbole und der Repräsentation zu sprechen, denn in solchen Begriffen bleibt allemal jenes generierend Signifikatorische dominant, das die Signifikate zum sekundären Schein macht«(S.86 Anm. 124). Dieser Schluß stellt jedoch eine schwer begründbare Verallgemeinerung dar. ${ }^{19} \mathrm{Um}$ beim Beispiel zu bleiben, so ist nicht zu übersehen, daß die Identität von präsenter Hostie und absentem Gott, also nicht die Repräsentation, sondern die Realpräsenz Gottes,

S. $64 \mathrm{ff}$. et pass.), am traditionsmächtigsten darunter die vera icon der heiligen Veronika. Gerade die Gemälde mit dem Abbild Veronikas und dem ungemalten >Bild Christi, dem sog. Schweißtuch, böten übrigens einprägsame Beispiele für das, was Czerwinski die Aggregation völlig divergenter Ordnungen - hier eines Raumes der Zeichendifferenz und eines Raumes der ,Gegenwärtigkeit - nennt.

${ }^{18} \mathrm{Vgl}$. auch meine Rezension von: Hedda Ragotzky / Horst Wenzel (Hg.), Höfische Repräsentation. Das Zeremoniell und die Zeichen. Tübingen 1990. In: IASL 18,1 (1993) S. 197-209.

19 Anders wäre Eucharistie in der modernen Welt der Zeichendifferenzen nicht denkbar. Sie ist es aber, zumindest noch in der zeitgenössischen Theologie. 
auf den eucharistischen Akt begrenzt und daß diese Eingrenzung liturgisch synästhetisch markiert wird, also zeichenhaft! Was für den eucharistischen Raum totaler Gegenwärtigkeit gilt, ist also gerade nicht verallgemeinerungsfähig, dieser ist überdeutlich als singulärer Sonderfall herausgehoben aus einer Welt auch der Differenzen und Vermittlungsleistungen. Ihr darf man schwerlich die uns geläufigen Zeichenstrukturen und -regeln als die selbstverständlichen unterstellen - dies mit Czerwinski -, es ist so aber auch nicht - dies gegen ihn - die Existenz von Zeichenstrukturen, Symbolen, Repräsentationen zu leugnen.

An den unterschiedlichen Gegenständen wäre dies nun im einzelnen zu entfalten: Die Repräsentationsdiskurse der Veronika-Legenden zum Beispiel, an jener des sogenannten Wilden Mannes könnte das etwa prägnant gezeigt werden, ${ }^{20}$ profilieren gerade die Gegenwärtigkeit des Heilands in der vera icon, indem sie diese von der Zeichenhaftigkeit der Bilder ${ }^{21}$ (etwa der Lukas-Portraits) absetzen. Die Präsenz von Unmittelbarkeit in einer historisch fremden Welt beweist für dort nicht die Absenz von Vermittlungen.

3. Ausschlußverhältnis von Gegenwärtigkeit und Zeichen: Hier wird nochmals jene problematische Strategie greifbar, mit welcher Czerwinski sein Projekt einer Historisierung der Zeichen durcharbeitet. Die Opposition des Begriffs der Gegenwärtigkeit zu dem des Zeichens wird - aufs Ganze des vorliegenden Buches gesehen - als Ausschlußverhältnis gefaßt. Der als Motto und auch sonst wiederholt zitierte Satz von Aleida Assmann: »Wo die Dinge gegenwärtig sind, gibt es keine Zeichen« (S. 7, 47, 272 Anm. 438), trägt hier die logisch unbegründete Bedeutung: Weil es im Mittelalter unvermittelte Gegenwärtigkeit gibt, gibt es im Mittelalter keine Zeichen. Dieser Schritt aber, >Gegenwärtigkeit und 'Zeichendifferenz< als generelle Wahrnehmungslogiken in eine Ausschlußrelation zu rücken, treibt jene Dichotomisierung der 'Welt überhaupt hervor, welche den Versuch radikaler Historisierung der mediävistischen Begriffe in der reduktionistischen Dehistorisierung des Mittelalters ${ }^{22}$ sich festfahren läßt. Mittelalterliche Wahrneh-

${ }^{20}$ Vgl. Die Gedichte des Wilden Mannes. Hg. von Bernard Standring. (ATB 59) Tübingen 1963, hier S. $1-30$.

21 Es ergeben sich also nicht nur argumentationslogische, sondern auch vom Material her Einwände gegen die überaus zugespitzte These: »die Struktur der Generation eines dann natürlich sekundären - Ab-Bildes durch ein - primäres - Ur-Bild gehört genuin [!] und allein [!] zur bürgerlichen Gesellschaft und trifft die historische Besonderheit mittelalterlicher Dinglichkeit / Körperlichkeit nicht [...]《 (S. 271). Es ist der Preis solcher Pointierung, daß immer wieder überlieferte mittelalterliche Differenzierungsleistungen - eklatant z. B. S. $38 \mathrm{f}$. bei einer Kommentierung des Iwein-Prologes - im blinden Fleck der hier durchgeführten Konzeption liegen.

${ }^{22}$ Ein Indiz dafür ist vielleicht auch, daß gegenüber denen im vorangegangenen die $\mathrm{Da}$ tierungen im vorliegenden Band deutlich indirekter geworden sind: Zeitliche Fixierungen etwa für dieselben narrativen Großformen erfolgen nicht mehr kalendarisch (»Beginn des 13. Jahrhunderts", »um 1200«, Der Glanz der Abstraktion, S. 12,15 usw.), sondern überwiegend metaphorisch: "Territorialisierung (S. 293, etwa auch S. $244 \mathrm{ff}$,, 262, 266 Anm. 426, 279f., 284, 305 et pass., 394, 416); vgl. auch hier Anm. 14, 24. 
mungsstrukturen werden nicht mehr mit dem Instrumentarium anachronistischer bürgerlicher Terminologien kolonisiert, sie gehen jetzt im Gegenteil in der Universalität einer archaischen Vor-Zeit auf. Denn im Prinzip hat die Welt nur noch diese beiden einander ausschließenden Teile: den unsrigen und den anderen. ${ }^{23}$ Es gibt zum Beispiel neben den »beiden grundsätzlichen Arten, den Raum zu organisieren, der bürgerlichen und der nicht-bürgerlichen « (S. 58), keine Alternativen, auch keine Übergangszonen. Noch die Berührung des einen mit dem anderen Paradigma geschieht nach dessen nichtbürgerlichen Regeln aggregativ, noch der Wechsel von dort nach hier ist ein Sprung (also: nicht sukzessiv, kontinuierlich, linear).

In einem solchen Entwurf ist historischer Wandel, etwa der vom Mittelalter zur Neuzeit, nicht denkbar, ihm bleibt die Entstehung des Neuen das schlechthin Unerklärliche. Es kann allenfalls metaphorisch gefaßt werden, etwa als »Einbruch eines symbolischen Systems in die Unmittelbarkeit des noch ganz im Sein versenkten Bewußtseins « (S. 311). ${ }^{24}$ Nicht einholbar ist im Rahmen von Czerwinskis Konzeption aber auch etwa das Lachen ${ }^{25}$ oder die Lüge; sie kommt deswegen nur in indirekten Spiegelungen vor. ${ }^{26}$ Insgesamt kann hier Historisierung schwer-

${ }^{23}$ Meine Wiedergabe ist hier nicht ohne Pointierung. Historisch notwendige Differenzierungen werden von Czerwinski nicht einfach ignoriert, schon die Komplementarität der Exempel einer Geschichte der Wabrnebmung ist daraufhin angelegt: Stellte der erste Band dar, »wie um 1200 eine Welt unmittelbarer Präsenz in mehrere, symbolisch vermittelte Schichten zu zerfallen beginnt«, so will der zweite gegenläufig dazu das »Fortwirken [] einer nicht-kausalen, nicht-sukzessiven, nicht-systematischen Logik bis in die Neuzeit hinein « demonstrieren (S. 7 f.). Doch werden diese, die äußere Anlage der beiden Bände bestimmenden, historischen Verschränkungen gegenläufiger Prozesse nicht konzeptionell wirksam. Sie sind (vgl. dazu das folgende) in der prinzipiellen Dichotomie des Entwurfes nicht anders konzipierbar denn nach den Regeln der snicht-bürgerlichen Welt< als Aggregat von Aggregat und System. Und in der konkreten Arbeit am Material erscheint die Markierung historischer Differenzierungen und Übergänge auch als Form, ihre fälligen konzeptionellen Konsequenzen zu neutralisieren (exemplarisch etwa S. 379 bei der Diskussion der Kategorien 'Gewalt< und 'Strafe $<$ ).

${ }^{24}$ Es gibt demnach auch präzise Epochengrenzen, welche von »historische[n] Umbruchslinie[n] « markiert werden, selbst wenn sie sich nicht datieren lassen sollten: »irgendwann [beginnt] eine virtualisierende Logik des Zeichens [...]." (S. 274); vgl. auch S. 5, 77, 81, 87, 93, 110 f., 266f., 311, 489, 502 usw.

${ }^{25}$ Das Lachen wird umstandslos als »versöhnende[s] Lachen des Bürgers « (S. 464) pauschalisiert zur Negation des nicht-bürgerlichen Gelächters. Doch liegen Differenzierungen auf der Hand: Vom Charivari bis zur bürgerlichen Ästhetik des 18. Jahrhunderts (etwa im 28. Stück von Lessings Hamburgischer Dramaturgie) ist VVernichtung. als eine Modalität auch bürgerlichen Lachverhaltens zu beobachten und also eine $\mathrm{Di}^{\mathrm{i}}$ chotomie hinfällig, nach welcher das »vernichtende Gelächter [...], das öffentliche, kollektive Funktion hatte und zumeist apotropäischen Charakter, das Abwehr (sozialen) Unheils sein sollte (ebd.), eine historisch abgeschiedene Form des Lachens sei, die mit der nicht-bürgerlichen Welt untergegangen wäre.

${ }^{26}$ Nämlich in der Kritik am mediävistischen Gebrauch des Fiktionalitätsbegriffs (vgl. besonders S. $266 \mathrm{ff}$;; vgl. auch Der Glanz der Abstraktion S. $281 \mathrm{ff}$. u. ö.), sodann bei der Besprechung von Fälschungen im Mittelalter (S. $229 \mathrm{ff}$.). Doch wiederum: Die berechtigte Insistenz auf der Historizität auch einer Kategorie wie derjenigen der histori- 
lich etwas anderes hervortreiben, als die (diatopisch, diachronisch, diastratisch) undifferenzierte Universalität des ahistorischen Anderen. Beobachtungen zur Kultur der Melanesier, der alten Ägypter, der Nordgermanen, Befunde an frühmittelalterlichen monastischen Traktaten, feudalhöfischen Romanen, ${ }^{27}$ akademischen Quaestionen, spätmittelalterlichen städtischen Bildern usw. stehen umstandslos füreinander ein und führen immer wieder auf das Eine: das universale Andere (welches seine Mitte dann aber letztlich doch in der Welt des hochmittelalterlichen deutschen Territorialadels findet, vgl. die Hinweise in Anm. 22). Zur Formel verkürzt begegnet dieses umfassend(e) Andere dort, wo immer wieder die Rede geht, eine fremde Wahrnehmungsform oder Wissensordnung sei "nicht-bürgerlich offenbar ubiquitär" (S. 235, 359, 390, 416, 433, 473 u. o.). Daß die Fremdheit fremder Ordnungen gerade auch in der inkommensurablen Andersheit ihrer Ausdifferenzierungsmuster zu finden sein mag, auch das ist in diesem Umriß schwer zur Geltung zu bringen.

4. Bestimmte Negation von Gegenwärtigkeit und Zeichen: In diesem Zusammenhang wird nun auch Czerwinskis Wortwahl signifikant: Das Andere heißt hier gerade nicht das >Andere, sondern das >Nicht-Bürgerliche<. Die Welt der Gegenwärtigkeit und die Welt der Zeichen schließen einander nicht nur einfach oppositiv (S. 7 Anm. 4) aus, sie sind zugleich im Verhältnis bestimmter Negation aufeinander bezogen. ${ }^{28}$ Hier liegt ein weiteres Zentralproblem des Entwurfs: Er wird durchgängig von einer starr zweistelligen Logik organisiert. Aus der Einsicht, daß für uns über die Fremdheit etwa >der mittelalterlichen Welt »keine positive, auf irgendeiner Anschauung beruhende Aussage mehr möglich" sei (S. 81), daraus wird gefolgert, daß diese fremde Welt allenfalls als bestimmte Negation der uns vertrauten Welt zulänglich begriffen werden könnte. Czerwinskis Rekonstruktionsstrategie zielt zurecht - wie man aus der Perspektive eines hermeneutischen Alteritätskonzeptes sagen wird - grundsätzlich auf »die geschichtliche Fremdartigkeit und Besonderheit ihres Gegenstandes« (S. 80). Zugleich bändigt sie indes die Verstörungspotentiale eines radikalen Alteritätskonzeptes sofort wieder, indem sie das Fremde, das Andere gerade nicht als dieses selbst, vielmehr als die bestimmte Negation bürgerlicher Epistemologien begreift. Dazu ist zwei-

schen Fälschung besagt noch nichts gegen die Möglichkeit der Lüge in der mittelalterlichen Welt, die indes schon damit keine der totalen Gegenwärtigkeit mehr wäre.

27 Freilich bestand im Mittelalter ${ }^{2} z$ wischen der weltlichen und der klerikalen Curialkultur jene Opposition nicht » (S. 314), die man allzu lange von der modernen Trennung von Staat und Kirche her dorthin projiziert hatte. Doch daß die Grenzen an den Stellen nicht liegen, wo sie selbstverständlich liegen müßten, impliziert nicht, es habe gar keine Grenzen gegeben. Ausdifferenzierungen von Wissensordnungen unterstellt Czerwinski andernorts ja auch selbst, dort etwa, wo er Spuren schamanistischer Praktiken liest (S. $329 \mathrm{ff}$. etc.).

${ }^{28}$ So schon Peter Czerwinski: Das Nibelungenlied. Widersprüche höfischer Gewaltreglementierung. In: Winfried Frey [u.a.], Einführung in die deutsche Literatur des 12. bis 16. Jahrhunderts. Bd. 1: Adel und Hof - 12./13. Jahrhundert. Opladen 1979, S. 4987. Hier S. 68. 
erlei zu bemerken: Erstens dies, daß >Alterität hier nicht als verstehenstheoretischer Begriff gebraucht wird, sondern als ontologische Setzung eines faktisch gegebenen, unüberwindbar tiefen kulturellen Hiatus zwischen dem Mediävisten und seinen Gegenständen. Anders als in der hermeneutischen Tradition und näherhin in der Mediävistik von Jauss bis Zumthor ${ }^{29}$ ist Alterität für Czerwinski nicht funktionale Regel eines auch in der Erwartung gelingenden Verstehens angestrengten hermeneutischen Prozesses, sondern substanzialistische Gegenstandskategorie. ${ }^{30}$ Alterität ist hier die Negation des Eigenen schlechthin.

So entsteht, zweitens, ein binäres Begriffsgebäude, das nicht nur Leittermini wie Sukzessivität und Simultaneität, Linearität und Zyklik, Abstraktion und Konkretion, Systematizität und Aggregativität einander zuordnet, sondern in vielfältigsten Negationspaaren den Entwurf Czerwinskis und seine Deutungsleistungen in allen Einzelheiten bestimmt. Bürgerlich vs. nicht-bürgerlich heißt also etwa auch: Relation vs. Substanz, reflexive Differenz vs. reflexionslose Einheit, Bedeutung vs. Sinn, Lektüre vs. Schau, Vermittlung / Zeichen / Repräsentation / Absenz vs. Gegenwärtigkeit, Denkform , Geschichte`vs. Denkform >Gegenwärtigkeit< (S. 235), System vs. Sichtbarkeit oder auch >Szene vs. Evidenz (S. 495), leere Transparenz vs. volle Evidenz, Kunst vs. Mythos (S. 361 ff.), Lachen vs. Gelächter (S. 464 ff.), usw. Die Welt überhaupt ist hier binär konstruiert. Sie wird in eine bürgerliche und eine nicht-bürgerliche Sphäre dichotomisch auseinandergelegt,

${ }^{29}$ Vgl. Hans Robert Jauss: Alterität und Modernität der mittelalterlichen Literatur. Gesammelte Aufsätze 1956-1976. München 1977, bes. S. 9 ff. In der Auseinandersetzung mit Jauss hat Paul Zumthor eine Ambiguität des Begriffs Alterität und zugleich einen Theorierest traditioneller Hermeneutik Diltheyscher Provenienz in dessen Voraussetzung einer unmittelbaren Zugänglichkeit der mittelalterlichen Welt und Literatur, bleibe sie auch präreflexiv, aufgedeckt. Zumthor hebt die ontologische »radical alterity « jeder Subjekt-Objekt-Beziehung ab von jener "relative alterity", die sich als Zuspitzung radikaler Alterität durch "varied circumstances", also "all concrete factors of a situation" einstelle (Paul Zumthor: Comments on H. R. Jauss's Article. In: New Literary History 10,2 [1979] S. 367-376. Hier S. 370, 376). Hieraus leitet er eine radikalisierende Umformulierung des methodologischen Prinzips ab, in welchem das mediävistische Alteritäts-Konzept kulminiert: »every text emanating from a distant epoch must, first of all, be received as a product of a universe in which we have no way of participating. Any analogy between this universe and ours must (until explicit proof of the contrary) be held as illusory «, woraus freilich Zumthor im Gegensatz zu Czerwinski nicht folgert, »that there are no pertinent analogies« (ebd., S. 370). Vgl. auch Paul Zumthor: Essai de poétique médiévale. (Collection Poétique) Paris 1972; Peter Haidu: Making it (New) in the Middle Ages. Towards a Problematics of Alterity. In: diacritics 4 (1974) S. 2-11; Alexandre Leupin: The Middle Ages, the Other. In: diacritics 13 (1983) S. 22-31; Rainer Warning: On the Alterity of Medieval Religious Drama. In: New Literary History 10,2 (1979) S. 265-292.

${ }^{30}$ Dies führt, beiläufig gesagt, in eine terminologische Paradoxie: In der substanzialistischen philosophischen Tradition ist Alterität nämlich Gegenbegriff zu Identität, Selbigkeit oder unitas, Einheit. Von hier her wäre, so müßte man mit Czerwinski sagen, die Alterität der mittelalterlichen Welt gerade ihre Identität, die Identität der bürgerlichen indes deren Alterität. 
und dieserart ist Alterität immer schon in einem Negationsverhältnis stillgestellt. Das Andere ist hier gar nicht das inkommensurable Fremde, als welches es von einem radikalen Alteritätskonzept gedacht werden müßte, sondern bloß die bestimmte Negation des Eigenen, des Vertrauten.

Immer wieder und immer wieder zu Recht klagt Czerwinski die Historisierung der Kategorien ein: »es gibt Oppositionen, - um dafür nun ein letztes Beispiel zu zitieren - »die haben nur bei der Analyse der bürgerlichen Gesellschaft einen Sinn. Die von 'Sein und Bewu\{tsein<//,Basis und Überbau</, Ökonomie und Ideologier gehören dazu, aber auch die von sheilig und profans, >Welt und Göttern«« (S. 63). Polemisch gesagt, gehört hierher auch die Opposition von >bürgerlich und nicht-bürgerlich<. Dabei muß es der Mediävistik in der Tat darauf ankommen, die historische Genese solcher Differenzierungen zu rekonstruieren. Doch gerade wie dies über das Konstrukt einer nivellierten Welt der totalisierenden Gegenwärtigkeit, der Nicht-Differenz als Negation sder bürgerlichen Welt der Differenzen gelingen könnte, vermag ich nicht zu erkennen. Erfolgversprechender könnte es vielmehr sein, die Gegenthese zu erproben, daß es in nichtbürgerlichen Welten diese Differenzen nicht so und an den Stellen gegeben habe, wo wir sie suchen, daß es sich nicht um Welten der totalen Nichtdifferenzierung, der totalen Gegenwärtigkeit handelte, sondern um Welten, die auf fremde (nicht einfach über bestimmte Negationen zu rekonstruierende) Weisen differenziert waren. Die Alterität der mittelalterlichen Welt auch in ihren Differenzen, Repräsentationen, Verweisungen, Ab-Bildern zu erarbeiten: das gelänge schwerlich über deren völlige Ausblendung.

V.

Es liegt in der Eigenart der von Peter Czerwinskis Vorstößen bewußt gemachten Herausforderungen der Mittelalterwissenschaften, der Grundsätzlichkeit der aufgeworfenen Probleme und auch der Aporien, in welche ihre Bearbeitung hier führt, daß sich die Diskussion im Einzelnen wie im Prinzipiellen stetig weiterführen ließe - die letzten der vorangegangenen Bemerkungen werden das zu erkennen gegeben haben. Fortsetzen wollen wird man ein solches kritisches Gespräch dabei gerade in dem Maße, in welchem man sich den erkenntnisleitenden Impulsen dieses Projektes verbunden fühlt und zumal dann, wenn man in theoretischen Grundsatzfragen wie in sachlichen Einzelproblemen immer wieder Alternativen für plausibler halten möchte. Fortgesetzt zu werden verdient die kritische Auseinandersetzung aber nicht zuletzt auch darum, weil der hier vorliegende Versuch einer radikalen Distanznahme gegenüber den disziplinären mediävistischen Konventionen des Lesens und Betrachtens ja auch diese unter Begründungsdruck setzt, und weil er im Falle dicht am Bild oder nah am Text geführter Analysen stets zu ganz neuen Wahrnehmungen führen kann; dieser Fall stellt sich freilich unter den Kohärenzwängen integraler Textanalysen im ersten Band der Exempel 
einer Geschichte der Wabrnehmung häufiger als im zweiten ein. Jedenfalls scheint es mir ausgemacht, daß es sich bei der Kritik von Peter Czerwinskis MittelalterEntwurf um ein ertragreiches Streiten handeln kann, und solches erkennbar werden zu lassen war nicht zum wenigsten die Absicht dieses Diskussionsbeitrages. 